\title{
CONSTRUCTIVE REFLEXIVITY OF A UNIFORMLY CONVEX BANACH SPACE
}

\author{
HAJIME ISHIHARA
}

(Communicated by John B. Conway)

\begin{abstract}
In this paper we consider a question about reflexivity of a Banach space within the framework of Bishop's constructive mathematics and we give a partially affirmative answer to the question set by Bishop: "Is every uniformly convex Banach space reflexive?".
\end{abstract}

In this paper we consider a question about reflexivity of a Banach space. Classically, the question has an answer that every uniformly convex Banach space is reflexive; see [6, Theorem 8.2.1; 7, Theorem V.2.2]. However, within the framework of Bishop's constructive mathematics, the question is more sensitive, because, for a bounded linear functional $f$ on a normed linear space $E$, the norm

$$
\|f\| \equiv \sup \{|f(x)|: x \in E,\|x\| \leq 1\}
$$

does not always exist and hence we cannot treat the dual space $E^{*}$ of $E$ as classically. In particular, we do not know that the set $E^{\prime} \subset E^{*}$ of normable linear functionals (i.e. linear functionals whose norms exist) on $E$ is a linear space. Therefore Bishop called a Banach space $E$ reflexive if

(A) $E^{\prime}$ is closed with respect to addition, that is, $E^{\prime}$ is a linear space;

(B) every normable linear functional on $E^{\prime}$ is of the form $f \mapsto f\left(x_{0}\right)$ for some $x_{0}$ in $E$, and set a question: "Is every uniformly convex Banach space reflexive?"; see $[1$, p. $295 ; 2$, p. 393$]$ and, for the foundations of constructive mathematics, see $[\mathbf{1}, \mathbf{2}, \mathbf{3}, \mathbf{4}]$. The main result (Theorem 2) of the present paper gives the question a partially affirmative answer: every uniformly convex complete normed linear space which satisfies the condition (A) is reflexive, that is, the condition (B) holds.

Let $\mathbf{F}$ denote either the real or the complex number field and let $E$ be a normed linear space over $\mathbf{F}$. Then $E$ is said to be uniformly convex if for each $\varepsilon>0$ there exists $\delta(\varepsilon)>0$, called the modulus of convexity of $E$, such that for each $x, y \in E$ with $\|x\| \leq 1$ and $\|y\| \leq 1$,

$$
\left\|\frac{x+y}{2}\right\| \leq 1-\delta(\varepsilon) \text { whenever }\|x-y\| \geq \varepsilon .
$$

Let $f$ be a bounded linear functional on $E$ and let $V$ be a linear subset of $E$. Then the norm of $f$ as a linear functional on $V$ is

$$
\|f\|_{V} \equiv \sup \{|f(x)|: x \in V,\|x\| \leq 1\}
$$

when it exists.

Received by the editors November 2, 1987.

1980 Mathematics Subject Classification (1985 Revision). Primary 46R02; Secondary 03F65.

Key words and phrases. Reflexive, uniformly convex, constructive. 
We begin with the following lemma which plays a crucial role in the proof of theorem.

LEMMA 1. Let $E$ be a normed linear space over $\mathbf{F}$ which satisfies the condition (A) and let $Y$ be a finite-dimensional subspace of $E^{\prime}$. Then for each $\varepsilon>0$ there exists a finite-dimensional subspace $V$ of $E$ such that

$$
\|f\|_{V} \geq(1-\varepsilon)\|f\| \quad(f \in Y) .
$$

ProOF. Let $S_{Y} \equiv\{f \in Y:\|f\| \leq 1\}$ and let $\eta$ be a positive number with $\eta(5+\eta)<\varepsilon$. Then since $S_{Y}$ is a compact subset of $E^{\prime}$ there exists an $\eta$ approximation $\left\{g_{1}, \ldots, g_{m}\right\}$ to $S_{Y}$. Also since each $g_{i}$ is normable, there exists $u_{i}$ in $E$ such that $\left\|u_{i}\right\|=1$ and $\left|g_{i}\left(u_{i}\right)\right|>\left\|g_{i}\right\|-\eta$. By Lemma 2.5 of $[2$, p. 308], there exists a finite-dimensional subspace $V$ of $E$ such that $d\left(u_{i}, V\right)<\eta$ for $i=1, \ldots, m$. Let $f$ be a linear functional in $Y$ with $\|f\| \leq 1$. Then there exists $i$ and $v_{i} \in V$ such that $\left\|f-g_{i}\right\|<\eta$ and $\left\|u_{i}-v_{i}\right\|<\eta$. Hence we have

$$
\begin{aligned}
\|f\| & \leq\left\|g_{i}\right\|+\left\|f-g_{i}\right\|<\left|g_{i}\left(u_{i}\right)\right|+\eta+\eta \\
& \leq\left|f\left(v_{i}\right)\right|+\left|g_{i}\left(v_{i}\right)-f\left(v_{i}\right)\right|+\left|g_{i}\left(u_{i}\right)-g_{i}\left(v_{i}\right)\right|+2 \eta \\
& \leq\|f\|_{V}\left\|v_{i}\right\|+\left\|g_{i}-f\right\|\left\|v_{i}\right\|+\left\|g_{i}\right\|\left\|u_{i}-v_{i}\right\|+2 \eta \\
& <\|f\|_{V}(1+\eta)+\eta(1+\eta)+\eta+2 \eta=\|f\|_{V}+\eta\|f\|_{V}+4 \eta+\eta^{2} \\
& \leq\|f\|_{V}+\eta\|f\|+4 \eta+\eta^{2} \leq\|f\|_{V}+\eta(5+\eta)<\|f\|_{V}+\varepsilon .
\end{aligned}
$$

For arbitrary $f \in Y$ and each positive number $t$, putting $f_{t} \equiv f / \max \{t,\|f\|\}$, we have $\left\|f_{t}\right\|<\left\|f_{t}\right\|_{V}+\varepsilon$ and hence $\|f\|<\|f\|_{V}+\varepsilon \max \{t,\|f\|\}$. Therefore, letting $t \rightarrow 0$, we obtain

$$
\|f\| \leq\|f\|_{V}+\varepsilon\|f\| .
$$

Next, we prove a constructive substitute for Helly's Theorem; see [6, Theorem 4.10.1; 7, Theorem IV.6.5].

THEOREM 1. Let $E$ be a normed linear space over $\mathbf{F}$ which satisfies the condition (A) and let $M$ be a positive number. Suppose that $f_{1}, \ldots, f_{n}$ are in $E^{\prime}$ and $c_{1}, \ldots, c_{n}$ are in $\mathbf{F}$. Then the following are equivalent:

(1) For each $\varepsilon>0$ there exists $x_{0}$ in $E$ such that $\left\|x_{0}\right\| \leq M+\varepsilon$ and $\left|f_{k}\left(x_{0}\right)-c_{k}\right|<$ $\varepsilon$ for $k=1, \ldots, n$;

(2) $\left|\sum_{k=1}^{n} \lambda_{k} c_{k}\right| \leq M\left\|\sum_{k=1}^{n} \lambda_{k} f_{k}\right\|$ for every $\lambda_{1}, \ldots, \lambda_{n}$ in $\mathbf{F}$.

PROOF. If (1) holds, then for each $\varepsilon>0$ there exists $x_{0}$ in $E$ such that $\left\|x_{0}\right\| \leq$ $M+\varepsilon$ and $\left|f_{k}\left(x_{0}\right)-c_{k}\right|<\varepsilon$ for $k=1, \ldots, n$. It follows that for any $\lambda_{1}, \ldots, \lambda_{n}$ in $\mathbf{F}$ we have

$$
\begin{aligned}
\left|\sum_{k=1}^{n} \lambda_{k} c_{k}\right| & \leq\left|\sum_{k=1}^{n} \lambda_{k} f_{k}\left(x_{0}\right)\right|+\left|\sum_{k=1}^{n} \lambda_{k}\left(c_{k}-f_{k}\right)\right| \\
& \leq\left\|\sum_{k=1}^{n} \lambda_{k} f_{k}\right\|\left\|x_{0}\right\|+\varepsilon \sum_{k=1}^{n}\left|\lambda_{k}\right| \\
& \leq(M+\varepsilon)\left\|\sum_{k=1}^{n} \lambda_{k} f_{k}\right\|+\varepsilon \sum_{k=1}^{n}\left|\lambda_{k}\right| .
\end{aligned}
$$


Since $\varepsilon$ is arbitrary, we conclude that (1) implies (2). Conversely, suppose that (2) holds and let $Y$ be a finite-dimensional subspace of $E^{\prime}$ such that $d\left(f_{k}, Y\right)<$ $\varepsilon /(3 n(M+\varepsilon))$ for $k=1, \ldots, n$. Then, by Lemma 1 , there exists a finite-dimensional subspace $V$ of $E$ such that

$$
\|g\|_{V} \geq \frac{M}{M+\varepsilon}\|g\| \quad(g \in Y) .
$$

Let

$$
K \equiv\{x \in V:\|x\| \leq M+\varepsilon\}
$$

and, for each $k=1, \ldots, n$, let $g_{k}$ be a functional in $Y$ with

$$
\left\|f_{k}-g_{k}\right\|<\frac{\varepsilon}{3 n(M+\varepsilon)}
$$

Then, since $K$ is compact and convex,

$$
D \equiv\left\{\left(g_{1}(x), \ldots, g_{n}(x)\right): x \in K\right\}
$$

is a totally bounded convex subset of $\mathbf{F}^{n}$ with the norm

$$
\left\|\left(\lambda_{1}, \ldots, \lambda_{n}\right)\right\| \equiv\left(\left|\lambda_{1}\right|^{2}+\cdots+\left|\lambda_{n}\right|^{2}\right)^{1 / 2} .
$$

Suppose that $d \equiv d(c, D)>\varepsilon / 3$ where $c \equiv\left(c_{1}, \ldots, c_{n}\right)$. Then, by the separation theorem [2, Theorem 4.3 of p. 336 or 5 , Theorem 2], there exists $\left(\lambda_{1}, \ldots, \lambda_{n}\right)$ in $\mathbf{F}^{n}$ such that

$$
\left|\lambda_{1}\right|^{2}+\cdots+\left|\lambda_{n}\right|^{2}=1
$$

and

$$
\operatorname{Re} \sum_{k=1}^{n} \lambda_{k} c_{k} \geq \operatorname{Re} \sum_{k=1}^{n} \lambda_{k} g_{k}(x)+d \quad(x \in K) .
$$

Since $K$ is balanced, we have

$$
\begin{aligned}
\operatorname{Re} \sum_{k=1}^{n} \lambda_{k} c_{k} & \geq \sup _{x \in K}\left|\operatorname{Re} \sum_{k=1}^{n} \lambda_{k} g_{k}(x)\right|+d \\
& =(M+\varepsilon)\left\|\operatorname{Re} \sum_{k=1}^{n} \lambda_{k} g_{k}\right\|_{V}+d=(M+\varepsilon)\left\|\sum_{k=1}^{n} \lambda_{k} g_{k}\right\|_{V}+d \\
& \left.\geq M\left\|\sum_{k=1}^{n} \lambda_{k} g_{k}\right\|+d \quad \text { (by our choice of } V\right) \\
& \geq M\left(\left\|\sum_{k=1}^{n} \lambda_{k} f_{k}\right\|-\left\|\sum_{k=1}^{n} \lambda_{k}\left(g_{k}-f_{k}\right)\right\|\right)+d \\
& \geq M\left(\left\|\sum_{k=1}^{n} \lambda_{k} f_{k}\right\|-\sum_{k=1}^{n}\left|\lambda_{k}\right|\left\|g_{k}-f_{k}\right\|\right)+d \\
& >M\left(\left\|\sum_{k=1}^{n} \lambda_{k} f_{k}\right\|-n \frac{\varepsilon}{3 n(M+\varepsilon)}\right)+d \\
& >M\left\|\sum_{k=1}^{n} \lambda_{k} f_{k}\right\|-\frac{\varepsilon}{3}+d>\left|\sum_{k=1}^{n} \lambda_{k} c_{k}\right|
\end{aligned}
$$


This contradiction implies $d(c, D) \leq \varepsilon / 3$. Hence there exists $x_{0}$ in $K$ such that $\left|g_{k}\left(x_{0}\right)-c_{k}\right|<\varepsilon / 2$ for $k=1, \ldots, n$. Therefore we have, for each $k=1, \ldots, n$,

$$
\begin{aligned}
\left|f_{k}\left(x_{0}\right)-c_{k}\right| & \leq\left|f_{k}\left(x_{0}\right)-g_{k}\left(x_{0}\right)\right|+\left|g_{k}\left(x_{0}\right)-c_{k}\right| \\
& <\left\|f_{k}-g_{k}\right\|\left\|x_{0}\right\|+\frac{\varepsilon}{2}<\frac{\varepsilon}{3 n(M+\varepsilon)}(M+\varepsilon)+\frac{\varepsilon}{2}<\varepsilon .
\end{aligned}
$$

COROLLARY 1. Let $E$ be a normed linear space over $\mathbf{F}$ which satisfies the condition (A) and let $F_{0}$ be a nonzero normable linear functional on $E^{\prime}$. Then for any $f_{1}, \ldots, f_{n}$ in $E^{\prime}$ and any $\varepsilon>0$ there exists $x_{0}$ in $E$ such that

(1) $\left\|x_{0}\right\| \leq\left\|F_{0}\right\|+\varepsilon$,

(2) $\left|f_{k}\left(x_{0}\right)-F_{0}\left(f_{k}\right)\right|<\varepsilon(k=1, \ldots, n)$.

PROOF. Apply Theorem 1 with $c_{k} \equiv F_{0}\left(f_{k}\right), k=1, \ldots, n$, and $M \equiv\left\|F_{0}\right\|$.

Now, we can prove our main result.

THEOREM 2. Let $E$ be a uniformly convex complete normed linear space over $\mathbf{F}$ which satisfies the condition $(\mathrm{A})$. Then $E$ is reflexive, that is, the condition (B) holds.

ProOF. Suppose that $F_{0}$ is a nonzero normable linear functional on $E^{\prime}$. We may assume without loss of generality that $\left\|F_{0}\right\|=1$. Let $f_{0}, f_{1}, \ldots$ be a sequence in $E^{\prime}$, where $\left\|f_{k}\right\|=1$ for all $k$ and $F_{0}\left(f_{k}\right)>1-1 / k$ for $k=1,2, \ldots$. Then for each positive integer $n$, applying Corollary 1 with $\varepsilon \equiv 1 / n$, we construct $x_{n}$ in $E$ such that

(1) $\left\|x_{n}\right\| \leq 1+1 / n$

(2) $\left|f_{k}\left(x_{n}\right)-F_{0}\left(f_{k}\right)\right|<1 / n(k=0, \ldots, n)$.

In particular, for each $N=0,1, \ldots$, we have

$$
\left|f_{N}\left(x_{n}\right)-F_{0}\left(f_{N}\right)\right|<1 / n \quad(n>N) .
$$

We show that $\left\{x_{n}\right\}$ is a Cauchy sequence. Let $\delta$ be a modulus of convexity of $E$ and, for each $\varepsilon>0$, choose a positive integer $N$ so that $3 /(N+1)<\delta(\varepsilon / 2)$. For all $n$ and $m$ with $m, n \geq N$, suppose that $\left\|x_{m}-x_{n}\right\|>\varepsilon$. Then we have

$$
\begin{aligned}
& \left\|\frac{x_{m}}{1+1 / N}\right\| \leq \frac{1+1 / m}{1+1 / N} \leq 1, \\
& \left\|\frac{x_{n}}{1+1 / N}\right\| \leq \frac{1+1 / n}{1+1 / N} \leq 1
\end{aligned}
$$

and

Hence we obtain

$$
\left\|\frac{x_{m}-x_{n}}{1+1 / N}\right\|>\frac{\varepsilon}{1+1 / N} \geq \frac{\varepsilon}{2} .
$$

or

$$
\left\|\frac{x_{m}+x_{n}}{2(1+1 / N)}\right\| \leq 1-\delta\left(\frac{\varepsilon}{2}\right)
$$

$$
\begin{aligned}
\left\|x_{m}+x_{n}\right\| & \leq 2\left(1+\frac{1}{N}\right)\left(1-\delta\left(\frac{\varepsilon}{2}\right)\right) \\
& <2\left(1+\frac{1}{N}\right)\left(1-\frac{3}{N+1}\right) \\
& =2\left(1-\frac{2}{N}\right) .
\end{aligned}
$$


On the other hand, we have

$$
\begin{aligned}
\left\|x_{m}+x_{n}\right\| & =\left\|f_{N}\right\|\left\|x_{m}+x_{n}\right\| \\
& \geq\left|f_{N}\left(x_{m}\right)+f_{N}\left(x_{n}\right)\right| \\
& \geq 2\left|F_{0}\left(f_{N}\right)\right|-\left|F_{0}\left(f_{N}\right)-f_{N}\left(x_{m}\right)\right|-\left|F_{0}\left(f_{N}\right)-f_{N}\left(x_{n}\right)\right| \\
& >2\left(1-\frac{1}{N}\right)-\frac{1}{m}-\frac{1}{n} \geq 2\left(1-\frac{1}{N}\right)-\frac{1}{N}-\frac{1}{N}=2\left(1-\frac{2}{N}\right) .
\end{aligned}
$$

This contradiction shows that $\left\|x_{m}-x_{n}\right\| \leq \varepsilon$ for all $m, n \geq N$. Hence $\left\{x_{n}\right\}$ is a Cauchy sequence. Let $x_{0}$ be its limit. Then we have

$$
1-\frac{2}{n}<F_{0}\left(f_{n}\right)+\left(f_{n}\left(x_{n}\right)-F_{0}\left(f_{n}\right)\right)=f_{n}\left(x_{n}\right) \leq\left\|x_{12}\right\| \leq 1+\frac{1}{n}
$$

and hence, letting $n \rightarrow \infty$, we obtain $\left\|x_{0}\right\|=\lim _{n \rightarrow \infty}\left\|x_{n}\right\|=1$. Also since $\left|f_{N}\left(x_{n}\right)-f_{0}\left(f_{N}\right)\right|<1 / n$ for all $n>N(N=0,1, \ldots)$, we have

$$
\left|f_{N}\left(x_{0}\right)-F_{0}\left(f_{N}\right)\right|=\lim _{n \rightarrow \infty}\left|f_{N}\left(x_{n}\right)-F_{0}\left(f_{N}\right)\right|=0 \quad(N=0,1, \ldots) .
$$

Let $f \in E^{\prime}$. Then for each $\varepsilon>0$, either $\|f\|<\varepsilon / 2$ or $0<\|f\|$; in the former case, we have

$$
\left|f\left(x_{0}\right)-F_{0}(f)\right| \leq\left|f\left(x_{0}\right)\right|+\left|F_{0}(f)\right| \leq 2\|f\|<\varepsilon .
$$

In the latter case, applying the above procedure with $f_{0} \equiv f /\|f\|$, we have a vector $y_{0} \in E$ such that $\left\|y_{0}\right\|=1$ and $f_{n}\left(y_{0}\right)=F_{0}\left(f_{n}\right)$ for $n=0,1, \ldots$. Suppose that $\eta \equiv\left\|x_{0}-y_{0}\right\|>0$. Then we have $\left\|\left(x_{0}+y_{0}\right) / 2\right\|<1-\delta(\eta)$, so $\left\|x_{0}+y_{0}\right\|<2(1-\delta(\eta))$. On the other hand, since $f_{n}\left(y_{0}\right)=F_{0}\left(f_{n}\right)$ for $n=0,1, \ldots$, we have

$$
\left\|x_{0}+y_{0}\right\| \geq\left|f_{n}\left(x_{0}+y_{0}\right)\right|=2 F_{0}\left(f_{n}\right)>2(1-1 / n) \quad(n=1,2, \ldots)
$$

and hence $\left\|x_{0}+y_{0}\right\|=2$. This contradiction implies that $y_{0}=x_{0}$. Therefore we obtain

$$
\left|f\left(x_{0}\right)-F_{0}(f)\right|=\|f\|\left|g_{1}\left(y_{0}\right)-F_{0}\left(g_{1}\right)\right|=0<\varepsilon .
$$

Thus, in either case, since $\varepsilon$ is arbitrary, we have $f\left(x_{0}\right)=F_{0}(f)$. For a nonzero normable linear functional $F$ on $E^{\prime}$, we denote by $\kappa(F)$ the vector of $E$ such that $\|\kappa(F)\|=\|F\|$ and $f(\kappa(F))=F(f)$ for all $f \in E^{\prime}[2$, p. 323, (3.23)]. Let $F$ be an arbitrary normable linear functional on $E^{\prime}$ and let $\left\{z_{n}\right\}$ be a sequence of vectors in $E$ such that

$$
z_{n} \equiv 0 \quad \text { if }\|F\|<1 / k \text { for all } k=1, \ldots, n
$$

and

$$
z_{n} \equiv \kappa(F) \quad \text { if }\|F\|>0 \text { for some } k=1, \ldots, n .
$$

Then $\left\{z_{n}\right\}$ is a Cauchy sequence which satisfies $\left|\left\|z_{n}\right\|-\|F\|\right|<1 / n$ and $\mid f\left(z_{n}\right)-$ $F(f) \mid \leq\|f\| / n$ for all $f$ in $E^{\prime}$. Let $z_{0}$ be the limit of $\left\{z_{n}\right\}$. Then $\left\|z_{0}\right\|=\|F\|$ and $f\left(z_{0}\right)=F(f)$ for all $f \in E^{\prime}$.

ACKNOWLEDGEMENT. The author would like to thank the referee for many useful comments and suggestions. 


\section{REFERENCES}

1. E. Bishop, Foundations of constructive analysis, McGraw-Hill, New York, 1967.

2. E. Bishop and D. Bridges, Constructive analysis, Springer-Verlag, Berlin, 1985.

3. D. Bridges, Constructive functional analysis, Pitman, London, 1979.

4. D. Bridges and F. Richman, Varieties of constructive mathematics, London Math. Soc. Lecture Notes, no. 97, Cambridge Univ. Press, 1987.

5. H. Ishihara, On the constructive Hahn-Banach theorem, submitted.

6. R. Larsen, Functional analysis, Marcel Dekker, New York, 1973.

7. K. Yoshida, Functional analysis, Springer-Verlag, Berlin, 1968.

DEPARTMENT OF INFORMATION SCIENCE, TOKyo Institute OF TEChNOLOGy, OHOKAYAMA, MEGURO-KU, TOKYO, 152 JAPAN 